\title{
RÁNULA RECIDIVANTE CON MARSUPIALIZACIÓN. A PROPÓSITO DE UN CASO Y REVISIÓN DE LA LITERATURA
}

\section{RECLINING RANULA WITH MARSUPIALIZATION. A PURPOSE OF A CASE AND REVIEW OF THE LITERATURE}

\author{
Encalada-Abad Carolina ${ }^{1}$, Sarmiento Lenín ${ }^{2}$, Rodríguez Lourdes ${ }^{3}$ \\ ${ }^{1}$ Odontóloga Universidad de Cuenca. Ecuador. Egresada Maestría en Investigación Médica, Universidad San Martín de \\ Porres, Lima. Perú \\ ${ }^{2}$ Cirujano Oral y Maxilofacial, Universidad San Francisco de Quito, Docente Titular Universidad Católica de Cuenca, Jefe \\ de Servicio Clínica de Especialidades Médicas Paucarbamba Cuenca. Ecuador \\ ${ }^{3}$ Investigador Centro de Micología. IMPAM, Facultad de Medicina, UBA-CONICET- Argentina. Docente titular, Facultad \\ de Odontología Universidad de Cuenca. Ecuador \\ *caroencalada119@hotmail.com
}

\begin{abstract}
Resumen
Esta patología toma su nombre por la similitud que presenta con la garganta de una rana. Es un quiste mucoso que se forma en el piso de la boca, involucrando usualmente a las glándulas salivales mayores. Este artículo presenta el caso de una lesión quística compatible con ránula en una paciente de 29 años de edad, la cual recidivó a la marsupialización en un período de 40 días.
\end{abstract}

Palabras clave: Ránula, quiste, marsupialización, recurrente.

\begin{abstract}
This disease takes its name from the similarity it shows with a frog's underbelly. It is a mucous cyst that forms on the floor of the mouth, involving the major salivary glands in most cases. This paper presents a case of a cystic lesion, compatible with ranula in a 29 years old female that recurred in a period of 40 days with marsupialization technique.
\end{abstract}

Key words: Ranula, cyst, excision, recurrence.

\section{INTRODUCCIÓN}

Se define a una ránula, como un mucocele que se forma en el piso de la boca, y usualmente involucra las glándulas salivales mayores. Los mucoceles son una de las formaciones benignas mas comunes en boca, por definición son cavidades con contenido mucoso.1Resulta de la acumulación de saliva con motivo de la degeneración del tejido, pudiéndose formar por fenómenos de extravasación o retención mucosa. ${ }^{2}$

Se pueden dar dos mecanismos de formación: a)Quiste por Extravasación: causado por la ruptura traumática del conducto excretor o del parénquima glandular, lo que produce una fuga al tejido conjuntivo con una reacción inflamatoria adyacente. ${ }^{2}$ La acumulación de moco en el tejido conectivo circundante, forma un pseudoquiste, que carece de cubrimiento epitelial ${ }^{3}$ y suele crecer deslizándose entre los músculos milohioideo y geniogloso, y ser disecable al aumentar de tamaño a nivel de la línea media atravesando el frenillo lingual, y b)Quiste por Retención: causado por la obstrucción del conducto excretor de una glándula salival menor con bloqueo del flujo salival y dilatación del conducto. ${ }^{2}$

Específicamente, la ránula se origina en el cuerpo de la glándula sublingual, mas comúnmente en las áreas profundas del mismo. Le sigue en frecuencia la formación de quistes de retención en la entrada de los conductos de Rivinus y en la entrada del conducto de Wharton de la glándula submandibular. $^{3}$

La ránula no es una patología común de la cavidad oral, ocurre de 1 a $10 \%$ de los casos y tiene una prevalencia de 0.2 casos en 1000 personas. ${ }^{4}$ Existe una predilección de sexo, siendo más afectadas las mujeres en una proporción de 1:1.4 en relación a los hombres. La ránula cervical, tiene una predilección por el sexo masculino de 1:0.74. ${ }^{5}$ Se reporta que es más común en la segunda década de vida. ${ }^{3,5}$ Se encuentran lateralmente en el piso de boca,afectando mayormente al lado 
izquierdo, en una proporción de 1:0.62. ${ }^{7}$

Para el diagnóstico de la ránula es útil el uso de exámenes imagenológicos como ecografía, Tomografía Axial Computada y Resonancia Magnética.

Se debe realizar un diagnóstico diferencial con el quiste dermoide de piso de boca, cuya localización suele ser en la línea media en relación con el músculo genihioideo. Esta diferencia se corrobora con el estudio histopatológico, ya que los quistes dermoides contienen un líquido espeso amarillogrisáceo, de consistencia pastosa, con células epiteliales nucleares y anucleares y láminas de queratina. En caso de rotura del quiste aparecen histiocitos, células multinucleadas gigantes, granulomas de cuerpo extraño y un componente inflamatorio. ${ }^{8}$

Para eliminar la lesión se han reportado cuatro técnicas quirúrgicas:

- La marsupialización

- La escisión únicamente de la lesión

- La escisión de la glándula salival

- La escisión tanto de la lesión como de la glándula.

Se puede mencionar también terapias de resección con láser $\mathrm{CO} 2$,crioterapia, inyección intraquiste con sustancias esclerosantes (OK-432), y toxina botulínica.

Los tratamientos que no incluyen remoción total de la glándula sublingual, como incisión y drenaje, escisión de la ránula y marsupialización, presentan altas tasas de recurrencia. $^{9}$

\section{REPORTE DEL CASO}

Paciente de sexo femenino, de 29 años de edad sin antecedentes médicos relevantes, se presenta a la clínica de diagnóstico de la Facultad de Odontología de la Universidad de Cuenca, refiere molestia a causa de una tumefacción indolora al lado izquierdo de la lengua desde hace 2 meses, que había aumentado de tamaño con el tiempo. Indica como únicos síntomas resequedad bucal y dificultad para alimentarse. Ante la historia odontológica, reporta colocación de prótesis parcial removible bimaxilar hace 4 meses, sin referir molestias por la misma.

Al examen clínico, se observa una tumefacción en el piso de boca de lado izquierdo, redondeada, delimitada, de aproximadamente $3 \mathrm{~cm}$ de largo por $2 \mathrm{~cm}$ de ancho, coloración rosada, de consistencia suave y fluctuante, con dolor a la digitopresión profunda. Sin más lesiones orales ni linfadenopatía cervical.(Fig. 1)

Al correlacionar los hallazgos clínicos, se diagnosticó el caso como "ránula", se indicó una ecografía de piso de boca (Fig. 2) la cual demostró una lesión quística simple de pared fina $30 \times 23 \times 18 \mathrm{~mm}$, con un volumen estimado en $6 \mathrm{cc}$ sin vascularidad interna, por lo que se diagnosticó el caso como "ránula".

La paciente fue intervenida con la técnica de marsupialización, que consistente en la extirpación de la pared superior de la ránula, y la sutura de la membrana quística a la mucosa del piso de boca para hacer que continúe con la cavidad bucal. ${ }^{10}$ Se trabajó con anestesia por bloqueo regional del lado afectado, se realizó una incisión lineal posteroanterior, se disecó la pared superior, accediendo al quiste, se drenó el contenido mucoso se tomó una muestra de tejido para el estudio histopatológico y posteriormente se suturó con puntos simples la mucosa del piso de boca con el revestimiento quístico.

Se envió para estudio histopatológico, pieza ovoidea multilobulada de aproximadamente $2.5 \mathrm{~cm}$ de largo por $3 \mathrm{~mm}$ de ancho, color marrón parduzca, consistencia blanda; empleando formalina al $10 \%$ como solución fijadora (Fig. 4). La pieza fue procesada y teñida con tinción hematoxilina-eosina. El examen microscópico revela una cavidad quística llena de moco y abundantes células inflamatorias, tapizada por un tejido de granulación, exhibiendo una cápsula de tejido conectivo denso, con acúmulos de adiposidad. Adyacente se encuentran acinos mucosos y células inflamatorias en el estroma glandular, sin signos de malignidad, reportando un diagnóstico de quiste por extravasación mucoide (Fig. 4).

La paciente recibió terapia antiinflamatoria (Meloxicam $15 \mathrm{mg}$ ) por tres días sin referir complicaciones inmediatas a la cirugía. Sin embargo, cuarenta días luego de la cirugía, la paciente regresa con la misma sintomatología, refiriendo sequedad bucal y dificultad para mover la lengua. Al examen clínico se observa asimetría en el piso de boca, con una tumefacción en el lado izquierdo de forma ovalada, de $4 \mathrm{~cm}$ de largo por $3 \mathrm{~cm}$ de ancho (Fig.5), que generaba a su vez asimetría facial (Fig.5). Suave y fluctuante a la palpación, indolora y de color similar a la mucosa adyacente. Se le realiza a la paciente una sialometría, de la que se obtiene como resultado 0,08 confirmando xerostomía.

Se interviene a la paciente por segunda vez para extirpación de la ránula y de la glándula sublingual ipsilateral, una técnica menos conservadora pero que presenta la menor recurrencia. Se anestesia por bloqueo regional del lado afectado, se realiza una incisión posteroanterior y se procede a la remoción del quiste y acinos glandulares (Fig.6) posteriormente se sutura con puntos simples. ${ }^{1}$ se medica a la paciente con terapia antiinflamatoria (Meloxicam 15mg) Se controla a la paciente a la semana, para retiro de puntos, se realiza seguimiento a la paciente a los 3 meses y no refiere recidiva luego de la segunda cirugía (Fig.7).

\section{DISCUSIÓN}

Histopatológicamente la ránula es producida por la extravasación de saliva desde una glándula sublingual deteriorada y no está rodeada de epitelio. ${ }^{11}$ Clínicamente se ve como una vesícula cuyo color va del rosado normal hasta un azulado ligero, resultante de la cianosis y congestión vascular de los tejidos y el carácter translucido del fluido acumulado, bien definida, suave e indolora, aunque en algunos casos existe dolor a la palpación profunda. ${ }^{5}$ No siempre se presentan 

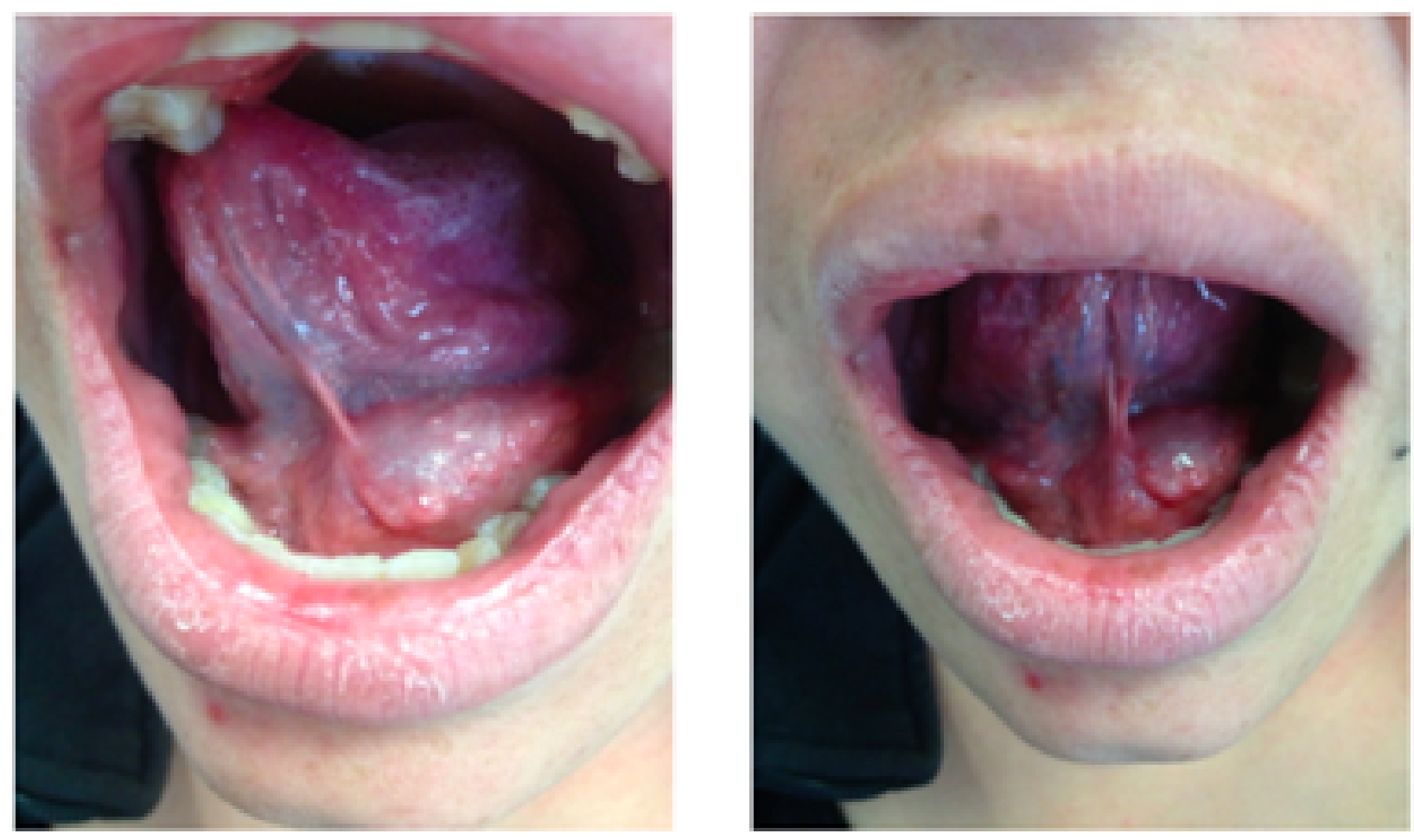

Fig. 1. Primera consulta: Tumefacción en el lado izquierdo del piso de boca.
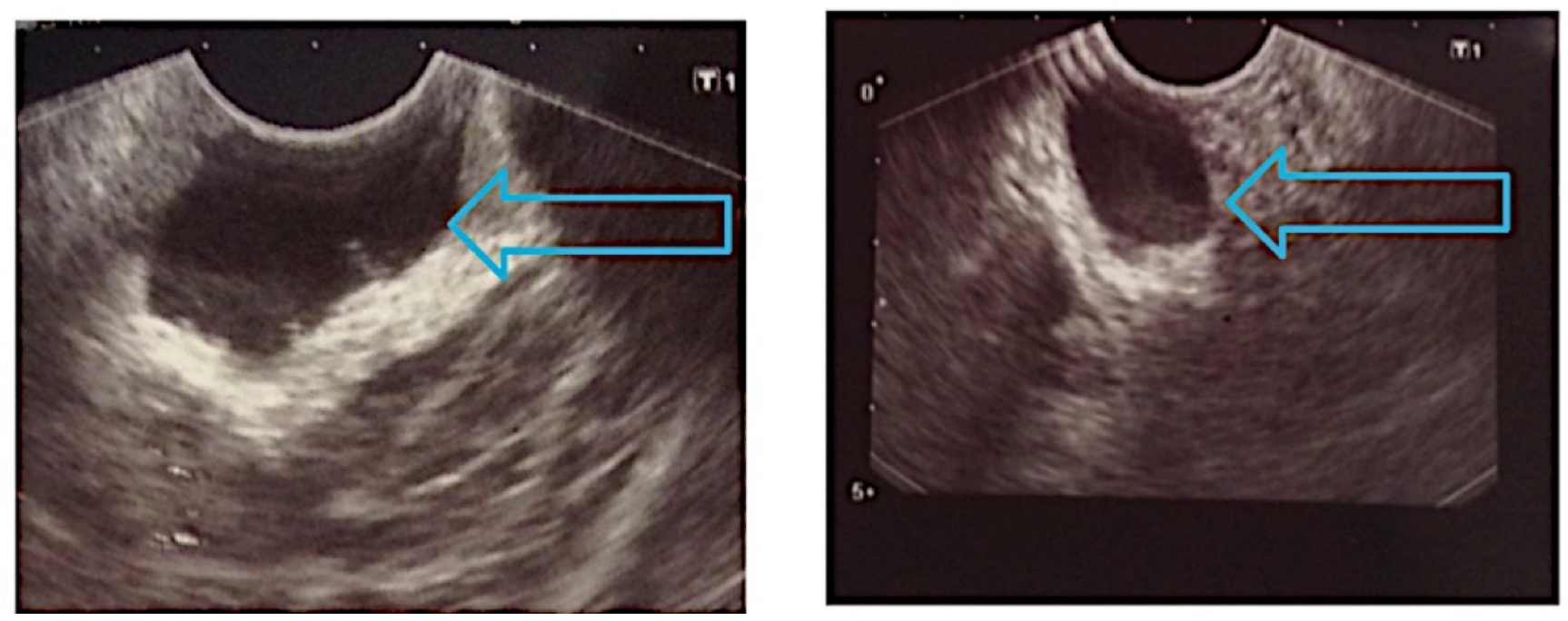

Fig. 2. Ecografía de partes blandas, región piso de boca. No se observa ni vascularidad ni obstrucción. 

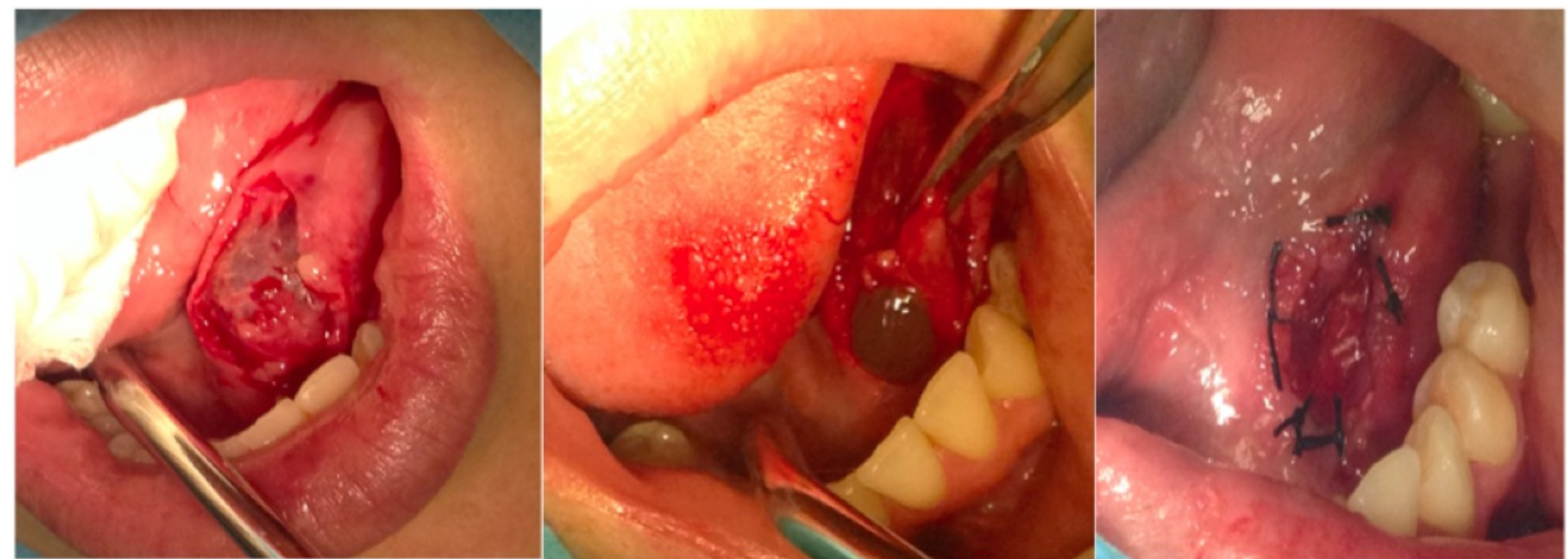

Fig. 3. Incisión lineal posteroanterior,3a. Disección de pared superior y presencia evidente de mucina. y 3b.Marsupialización.

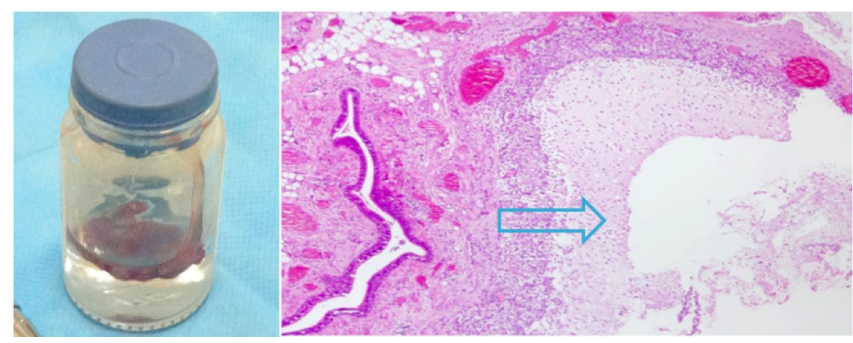

Fig. 4. Pieza quirúrgica para estudio Histopatológico. Corte de Estudio Histopatológico visto a 40x.
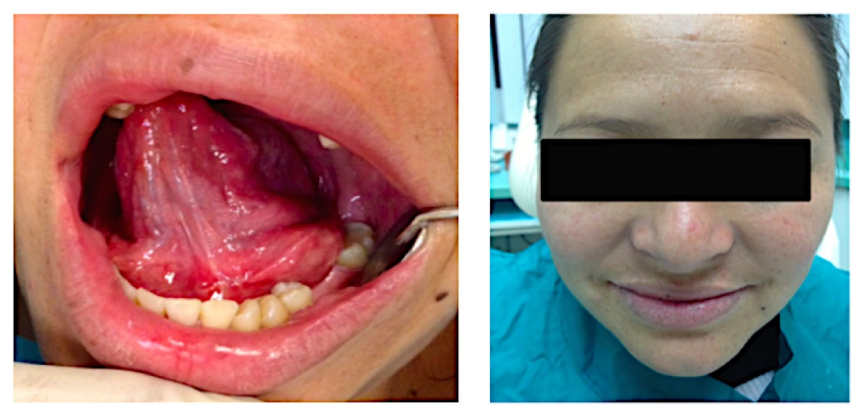

Fig. 5. Tumefacción en piso de la boca por reincidencia de ránula. Paciente mostrando ligera asimetría facial.

síntomas, pero puede causar incomodidad, interferencia al hablar, masticar o tragar. La ránula es una patología poco frecuente,tiene una prevalencia de 0.2 casos en 1000 personas. $^{4}$ En Cuenca-Ecuador, un estudio retrospectivo sobre patología quirúrgica en glándulas salivales, indica que la patología que con mayor frecuencia se presenta es el Tumor Mixto o Adenoma Pleomórfico de glándula Parótida. En cuanto a ránula la frecuencia es de un $6,6 \%$ reportando 2 casos en un

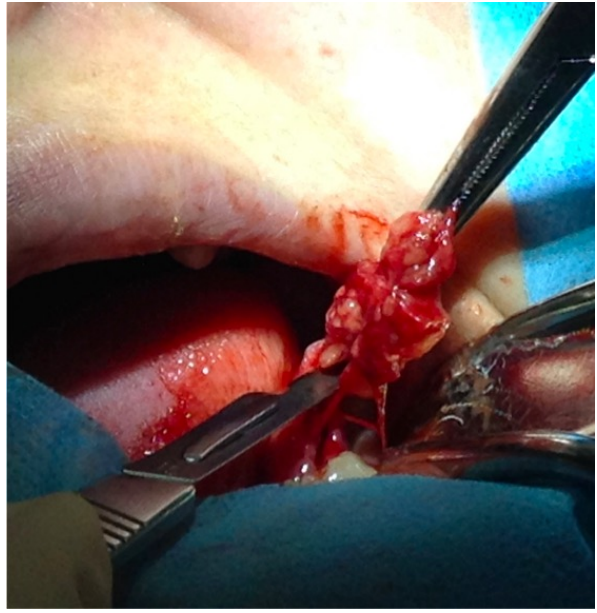

Fig. 6. Abordaje intraoral, remoción de quiste y acinos glandulares.

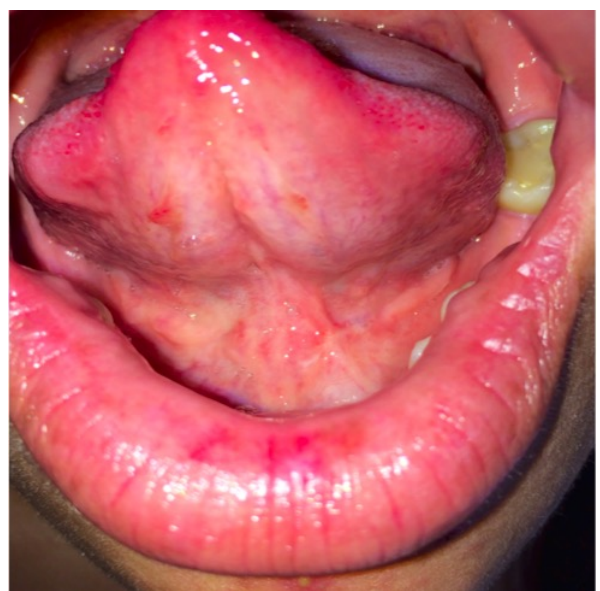

Fig. 7. Control postoperatorio a los 3 meses. 
periodo de 5 años. ${ }^{12}$

Como se mencionó anteriormente, para eliminar la lesión se han manejado técnicas como la marsupialización, escisión de la lesión, escisión de la glándula salival, o escisión tanto de la lesión como de la glándula.

Entre los principales problemas y complicaciones según la técnica quirúrgica se cita la recurrencia, relacionándola con la técnica de incisión y drenaje por que resulta en un cierre rápido de la herida y mayor probabilidad de recurrencia por llenado de fluido. La marsupialización a pesar de ser considerada la técnica de elección, es relacionada con altas tasas de recurrencia debido a que los márgenes de la herida suelen estar cercanos, sumado al movimiento de la lengua y el piso de boca, trae como resultado que se vuelva a formar la lesión entre 6 semanas y 12 meses con la posibilidad de extenderse a planos más profundos, y originar la llamada "ránula cervical". 9 La técnica de escisión total de la ránula y glándula, por su parte, presenta complicaciones como: sangrado, infección, daño al conducto de Wharton y parestesia del nervio lingual. ${ }^{6}$ La escisión de la ránula únicamente o la marsupialización, pueden ser útiles en casos de ránulas pequeñas, superficiales y protruyentes debido a que su origen de secreción es la porción superficial de la glándula sublingual y puede cerrarse al cicatrizar; en ránulas más profundas el caso será el contrario ya que tiene su origen en la porción central de la glándula sublingual y no sanará únicamente con la marsupializacion. ${ }^{13}$

Lee, D.H. y cols ${ }^{6}$ realizaron un estudio en 24 pacientes para determinar el tratamiento óptimo en casos de ránula. Incluyeron a 24 pacientes de los cuales 8 habían recibido tratamiento previo y recidivaron. Luego de las cirugías realizadas en el estudio, solo un caso presentó recurrencia ante la técnica de escisión total, por permanencia de residuo de pared quística. Los demás pacientes no presentaron recidiva, demostrando así que el abordaje intraoral con escisión de la ránula y glándula sublingual es el tratamiento de elección para evitar recurrencia en una ránula simple. ${ }^{13-15}$

El tratamiento estará a criterio del clínico, basándose en la edad del paciente y tamaño de la lesión; se recomienda marsupialización en lesiones pequeñas de difícil acceso y en infantes. ${ }^{4}$ Es importante mencionar la técnica de marsupialización modificada de Baurmash ${ }^{1}$ que consiste en aplicación de gaza en la cavidad para lograr compresión y cicatrización de la parte superficial de la herida y evitar la unión de los bordes de la misma, con un recurrencia de $12 \% .^{5} \mathrm{Se}$ han reportado también casos de ránula tratados con toxina botulínica tipo A. Una técnica poco invasiva, cuya eficacia no ha sido significativamente reportada. Como ventajas presenta la evasión de anestesia y trauma en el paciente, seguridad y tolerabilidad. El fármaco actúa por inhibición de las terminaciones nerviosas responsables de la salivación, se necesitan estudios de largo plazo para saber la recurrencia con este tratamiento. $^{16,17}$

Los niveles de recurrencia se relacionan íntimamen- te con la técnica quirúrgica, ${ }^{5}$ al comparar los porcentajes de recurrencia, la técnica de marsupialización presenta un $66.67 \%$; la recurrencia con escisión de la ránula es de $57.69 \%$ y la escisión total de glándula y lesión presentan recurrencia de $0 \%-1.2 \% .^{5}$ En un estudio retrospectivo de 12 pacientes se muestra una recurrencia de $16.7 \%$ a la marsupialización; $16.7 \%$ a la escisión del quiste y $0 \%$ de recurrencia en pacientes que se sometieron a escisión de la glándula ${ }^{4}$ lo que permite recomendarlo en este caso.

Conflicto de intereses y financiamiento Los autores declaran no tener conflicto de intereses, haber cumplido con los requisitos de autoría y haber autofinanciado este artículo.

\section{Referencias}

1 Baurmash HD. Mucoceles and ranulas. J Oral Maxillofac Surg. Mar; 2003 61(3):369-78.

2 Montagna F, Ferronato G, Martinelli F.Patologia orale orientata per problemi: diagnosi differenziale e terapia. Edición Promoass. Diciembre 2000; 132-133.

3 Jaishankar S, Manimaran, Kannan, Mabel C. Ranula A case report, JIADS Jul-Sept 2010; 1(3):50-53

4 Ghani NA, Ahmad R, Rahman RA, Yunus MR, Putra SP, Ramli R. A retrospective study of ranula in two centres in Malaysia. J of Maxilofac Surg. Nov 2002; 8(4):316-319.

5 Zhao YF, Jia Y, Chen XM, Zhang WF. Clinical review of 580 ranulas. Oral Surg Oral Med Oral Pathol Oral Radiol Endod 2004; 98:281-87.

6 Lee DH, Yoon TM, Lee JK, Lim SC. Treatment outcomes of the intraoral approach for a simple ranula. Oral Surg Oral Med Oral Pathol Oral Radiol. Apr 2015;119(4):223-5.

7 Kim, P.D., Simental, A. Salivary Gland Disorders. Springer Berlín Heidelberg. 2007:178-183.

8 Lin HW, Silver AL, Cunnane ME, Sadow PM, Kieff DA. Lateral dermoid cyst of the floor of mouth: unusual radiologic and pathologic findings. Auris Nasus Larynx. 2011;38(5):6503.

9 Zhao YF, Jia J, Jia Y.Complications Associated With Surgical Management of Ranulas J Oral Maxillofac Surg 2005; 63(1):51-4.

10 Leyva M. Lahera J, Díaz D, Pérez O. Ránula del suelo de la boca. A propósito de un caso. Revista Habanera de Ciencias Médicas 2005; 4(3):1-8.

1 Bhaskar SN, Bolden TE, Weinmann JP. Pathogenesis of mucoceles. J Dent Res Dec1956; 35(6):863-74.

12 Nunez del Arco, L. Patología quirúrgica de glándulas salivales en fundación otorrinolaringología Remigio Serrano Macías y Centro Quirúrgico Metropolitano Enero 2007Diciembre 2012 Documento disponible

13 Morita Y, Sato K, Kawana M, Takahasi S, Ikarashi F. Treatment of ranula-excision of the sublingual gland versus marsupialization. Auris Nasus Larynx. 2003; 30:311-314

14 Yang Y, Hong K. Surgical results of the intraoral approach for plunging ranula. Acta Otolaryngol. 2014;134:201-205. 
15 Huang SF, Liao CT, Chin SC, Chen IH. Transoral approach for plunging ranula-10 year experience. Laryngoscope. 2010;120:53-57.

16 Chow TL, Chan SW, Lam SH. Ranula successfully treated by botulinum toxin type A: report of 3 cases. Oral Surg Oral Med Oral Pathol Oral Radiol Endod 2008;105:41-42.

17 Vargas H, Galati LT, Parnes SM. A Pilot Study Evaluating the Treatment of Postparotidectomy Sialoceles With Botulinum Toxin Type A. Arch Otolaryngol Head Neck Surg. 2000;126(3):421-424.

Recibido: 04 de Julio de 2016

Aceptado: 18 de Julio de 2016 